\title{
MEMBRANE INDUCED OSTEOGENESIS (MASQUELET TECHNIQUE) VERSUS BONE TRANSPORT IN MANAGEMENT OF LARGE BONE DEFECTS OF THE LOWER LIMB
}

\author{
Salah Abdelgawad Abou-Seif, Mootaz Fouad Thakeb, Ahmed Hassan Yousry, \\ Mahmoud Ali Mahran, Tamer Abd El Meguid Mohamed Fayyad, and \\ Mohamed Ismail Abdel hakim Mohamed Kotb
}

\author{
Orthopaedic Department, Faculty \\ of medicine, Ain Shams \\ University. Cairo, Egypt \\ Corresponding: \\ Mohamed Ismail Abdelhakim \\ Mobile : 01112590513 \\ E mail: \\ mkotb37@gmail.com \\ Received: $23 / 1 / 2020$ \\ Accepted: 24/2/2020
}

Online ISSN: 2735-3540

\begin{abstract}
:
Background: Management of bone defects is controversial, between grafting, acute shortening, and up to amputation and disarticulation in extensive defects. This study is a comparative study between 2 modalities for management of bone defects to reveal advantages and disadvantages of each modality.
\end{abstract}

Aim of the work: The aim of this work is to assess and compare results of membrane induced osteogenesis and bone transport in extensive bone defects of the lower limb.

Patients and methods: We prospectively studied 40 patients (29 males and 11 females) with lower limb bony defects. They were divided into two groups according to the method of reconstruction using either Masquelet technique in 20 patients (group A) or bone transport in the other 20 patients (group B). The mean age of the patients was 35. $r$ years in group $A$ and was 35.2 years in group $B$. diagnosis was infected non union (20 cases: 10 in group $A$ \&10 in group B) and posttraumatic and non infective defects (20 cases: 10 in group A \&10 in group B). The most common anatomical site was the tibia (24 cases:9 in group A \&15 in group B).. Patients were reviewed every 3 monthly for a minimum period of 1 year. Regular outpatient follow-up was done with a mean period of $18.35 \pm 5.58$ months in group A and 18.25 \pm 3.95 months in group B.

Results: In cases of group A (Masquelet group) 12 cases showed complete union, and the other 8 cases showed no evidence of union (all from infected group). While in case of group B (bone transport group) all cases showed complete union

Conclusion: bone transport technique is more reliable method with more expectable results for healing and infection eradication. Up to our study, Masquelet technique can be used in the following indications: small, metaphyseal and posttraumatic or non infective defects

Keywords: Masquelet, bone defects, segment transfer, induced membrane, distraction osteogenesis.

\section{INTRODUCTION:}

Reconstruction of extensive bone defects of the limbs is still a major challenge, both for anatomical and functional results. The limits of traditional bone graft techniques are now clear, mainly because of uncontrollable graft resorption, even when the recipient site is well vascularized $^{1}$ 
The major technical options for the reconstruction of traumatic diaphyseal bone loss are traditional bone grafting (in the leg including the intertibiofibular graft and tibialization of the fibula), vascularized bone grafts, the induced membrane technique and bone transport techniques ${ }^{2}$.

In 1986, (A.C. Masquelet) conceived and developed a technique for large bone defects, based on the principle of the induced membrane ${ }^{3}$.

The principle of the induced membrane technique involves provoking a reaction to a foreign body by placing a cement spacer in the bone defect. The membrane induced by this foreign body is in fact a biological chamber which prevents graft resorption by providing vascularization and growth factors, as shown by various clinical, experimental and basic studies ${ }^{4}$.

This characteristic has been used to good effect in periprosthetic infections where staged reconstruction has been shown to be safe after removal of the spacer ${ }^{5}$.

Bone transport or Distraction osteogenesis (DO) is a surgical technique in which the intrinsic capacity of bone to regenerate is being harnessed to lengthen bones or to replace large segments of bone. It consists of the application of an external fixator to the affected bone, followed by an osteotomy of the bone and then gradual and controlled distraction is applied to the two bone segments ${ }^{6}$.

The histological features of distraction osteogenesis closely resemble those of fracture healing,. Immediately after the osteotomy, a hematoma is formed. As distraction progresses, this hematoma is organized into fibrous and fibrocartilaginous tissue ${ }^{7}$

\section{AIM OF THE WORK:}

The aim of this work is to assess and compare results of membrane induced osteogenesis and bone transport in extensive bone defects of the lower limb as regards functional, radiological outcomes and possible complications.

\section{PATIENTS AND METHODS:}

We prospectively studied 40 patients (29 males and 11 females) with lower limb bony defects. They were divided into two groups according to the method of reconstruction using either Masquelet technique in 20 patients (group A) or bone transport in the other 20 patients (group B).

Inclusion Criteria: both sexes., 18-60 yrs old, tibial or femoral bone defects $>4$ cm. Exclusion Criteria: Pathological and congenital defects, Defects less than $4 \mathrm{~cm}$, Multiple defects and Pediatric age group.

The mean age of the patients was 35.2 years in group A and was 35.2 years in group B. diagnosis was infected non union (20 cases: 10 in group A \& 10 in group B) and posttraumatic and non infective defects (20 cases:10 in group A \& 10 in group B) . The most common anatomical site was the tibia (24 cases: 9 in group A \&15 in group B)

Clinical Evaluation: History: Age, Sex, Occupation, Address, Special Habits, Complaint, H.P.I, Pain:

Examination: The condition of the overlying skin: whether freely mobile, adherent or presenting a discharging sinus related to the defect site. For the distal part of the limb: Careful examination of the affected limb. Distal pulsations and neurology (sensory and motor supply) of limb should be examined and documented.

Radiological Evaluation: Plain X-ray long film showing joint above and joint below.

Laboratory Investigations: ESR , CRP and $\mathrm{CBC}$. 
A. Pre-operative: Detailed consent was taken from the patient and his relatives, which contained the risks and the importance of the procedure, the need for follow up. Counselling was done with the patient and his family, in the presence of the multidisciplinary team of orthopedic limb reconstruction surgeon, plastic surgeons and radiologists.

B. Operative: The first step includes a large excision of infected or nonviable tissue and sequestrated bone using bone nibbler, drill bits, reamers and power saw .until (Paprika sign) is noticed indicating healthy bleeding bone is reached,medulla is also opened in both ends . complete removal of

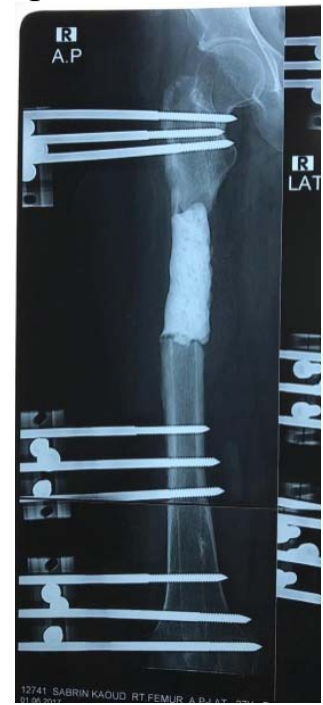

unhealthy bone is also confirmed by $\mathrm{C}$ arm. The cavity was copiously irrigated normal saline to remove any debris then in case of Masquelet tecnique a cement spacer and external fixator will be applied for 6-8 wks until inflammatory markers become normal then re operation by incision of the formed membrane, removal of cement spacer and filling the defect by cancellous graft with or without fibular graft and artificial bone and in case of bone transport external fixator will be applied and corticotomy will be done then distraction starts about 1 week later at a rate $1 \mathrm{~mm}$ per day, then the consolidation phase starts which counts about 1 month per $\mathrm{cm}$ distracted .

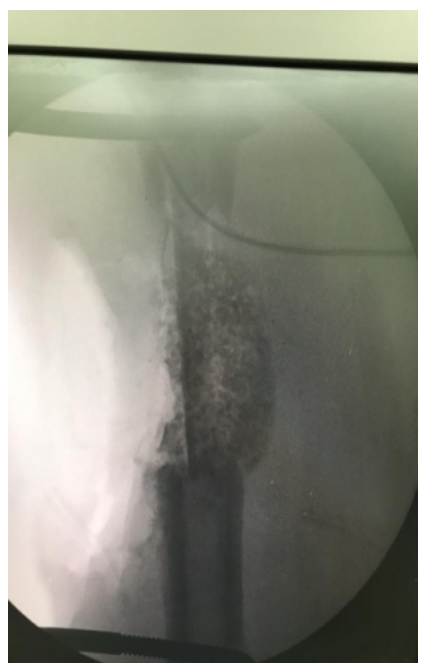

Figure 1: plain radiograph showing proximal femoral defect with cement spacer and after spacer removal and filling the defect with graft (from our study)

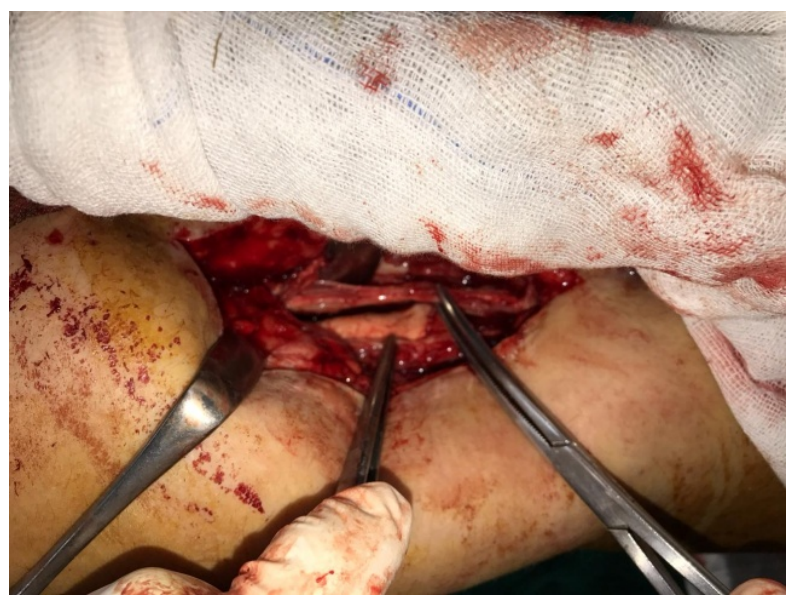

Figure 2 : the induced membrane and the cement spacer in Masquelet technique (from our study) 

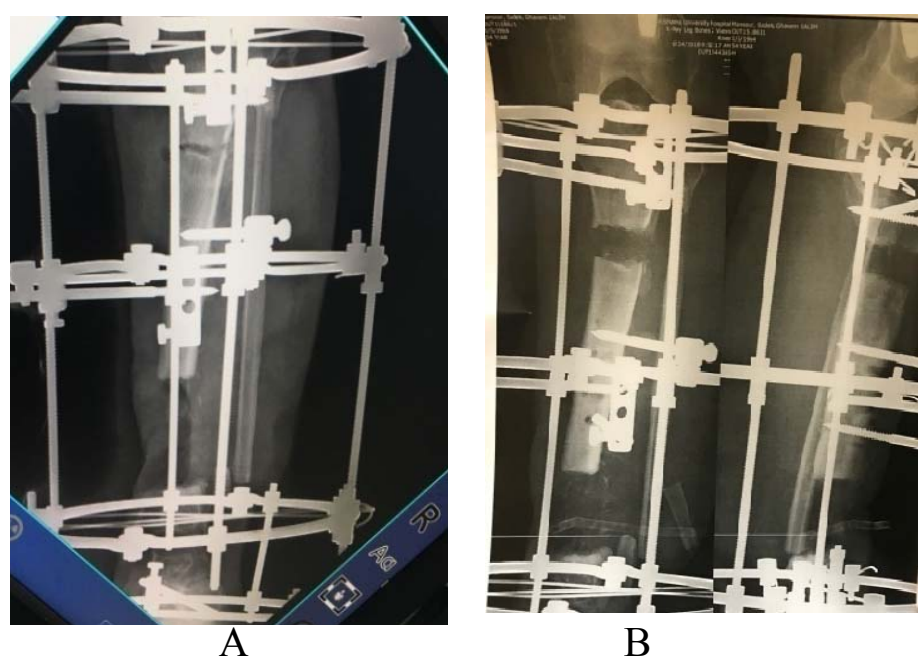

Figure 3: plain radiograph showing distal tibial defect with proximal corticotomy (A) and during distraction phase (B) (from our study)

C. Post-operative: Culture and sensitivity of the removed debris was done routinely, empirical antibiotic and sedation in the first few days postoperatively. Until the result of culture appears They were given intravenous infusion of fluids, to maintain good hydration. Hemoglobin levels were kept above $9 \mathrm{~g} / \mathrm{mL}$, postoperative $\mathrm{X}$-ray films were done in at least two views. Patients were given prophylaxis against deep venous thrombosis (DVT). The average hospital stay postoperative was 3 days, then patient will be discharged on the proper antibiotic according to culture .The patients were followed up in the out-patient clinic at regular intervals; weekly for 2 weeks, twice per month for the next 6 weeks, then monthly till the $6^{\text {th }}$ month, then every 3 months till the end of the $1^{\text {st }}$ year, and then twice per year. All of the patients were encouraged to begin partial weight bearing in the first postoperative month. Gradual improvement in weight bearing force was observed through X-ray findings during the follow ups. Passive range-of-motion training immediately after the surgery. Orthoses for extremities were used for not more than 3 months.

For patients of group B, patient is educated to start distraction after 1 week to 10 days at a rate of $1 \mathrm{~mm}$ per day divided into 4 times daily until reaching the docking site

\section{RESULTS:}

A. The Radiological results According to healing status assessed by plain x-ray and CT scan if needed .. accordingly cases are classified into :completely united, incompletely united, and no evidence of union. And according to ASAMI bone score.

Table 1 ASAMI scoring system for evaluation of the bone results.

\begin{tabular}{|l|l|}
\hline Bone results & \multicolumn{1}{c|}{ Criteria } \\
\hline Excellent & Union, no infection, deformity $<7^{\circ}$, limb length discrepancy (LLD) $<2.5 \mathrm{~cm}$ \\
\hline Good & $\begin{array}{l}\text { Union plus any two of the following: absence of infection, deformity }<7^{\circ}, \text { LLD } \\
<2.5 \mathrm{~cm} .\end{array}$ \\
\hline Fair & $\begin{array}{l}\text { Union plus any one of the following: absence of infection, deformity }<7^{\circ}, \text { LLD } \\
<2.5 \mathrm{~cm}\end{array}$ \\
\hline Poor & Nonunion/refracture/union plus infection plus deformity $>7^{\circ}$ plus LLD $>2.5 \mathrm{~cm}$ \\
\hline
\end{tabular}




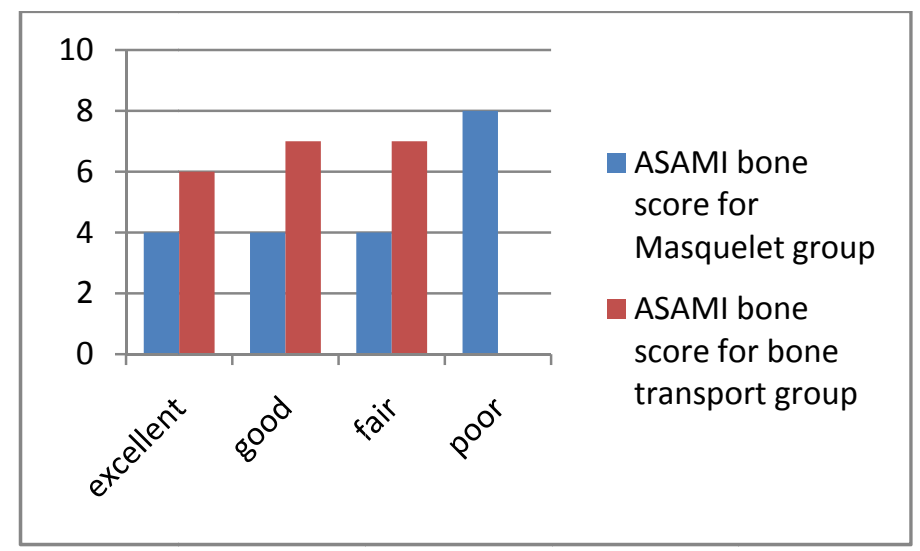

Diagram 1: bar chart for ASAMI bony score for both groups

B. The clinical and functional results : Clinical assessment of the patients includes recurrence of infection (in the form of discharging sinus or pintract infection) and wound dihescence. and according to ASAMI functional score

Table 2 :ASAMI scoring system for evaluation of the functional results.

\begin{tabular}{|l|l|}
\hline \multicolumn{1}{|c|}{ Functional results } & \multicolumn{1}{c|}{ Criteria } \\
\hline Excellent & $\begin{array}{l}\text { Active, no limp, minimum stiffness (loss of }<15^{\circ} \text { knee extension/ loss of }<15^{\circ} \\
\text { ankle dorsiflexion), no reflex sympathetic dystrophy (RSD), insignificant pain. }\end{array}$ \\
\hline Good & Active, with one or two of the following: limp, stiffness, RSD, significant pain \\
\hline Fair & Active, with three or all of the following: limp, stiffness, RSD, significant pain \\
\hline Poor & Inactive (unemployment or inability to return to daily activities because of injury) \\
\hline Failure & Amputation \\
\hline
\end{tabular}

In previously infected cases :In cases of group A ( Masquelet group) 4 cases showed no recurrence of infection, 6 cases showed recurrent infection. While in case of group B (bone transport group) 9 cases showed no recurrence of infection, 1 case showed recurrent infection.

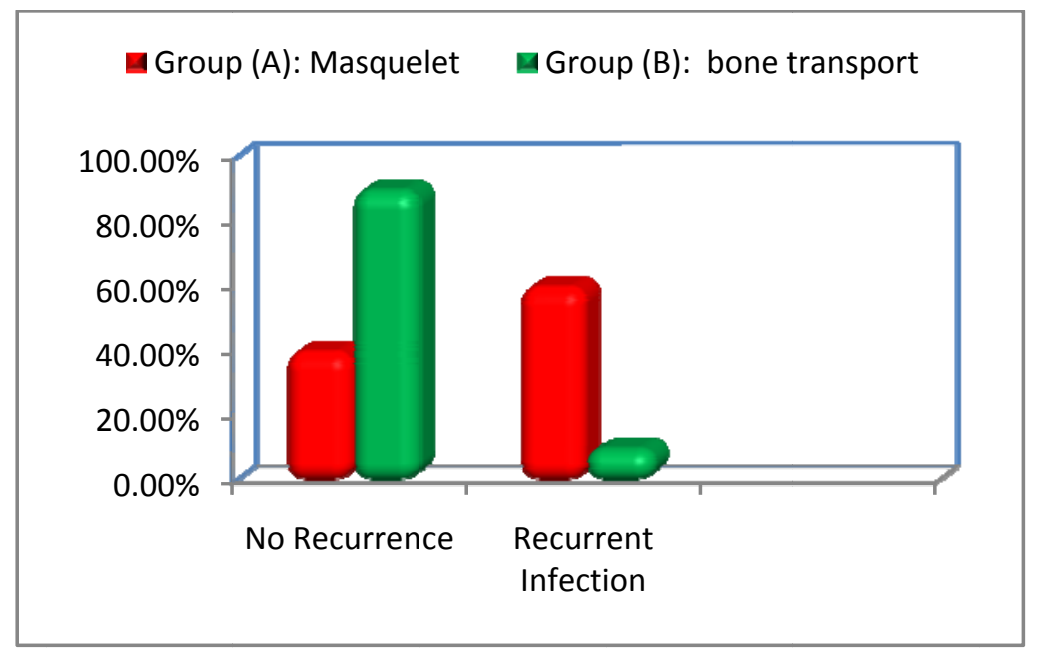

Diagram 2: Bar chart between group (A): Masqueletand group (B):bone transport according to recurrence of infectiopn 


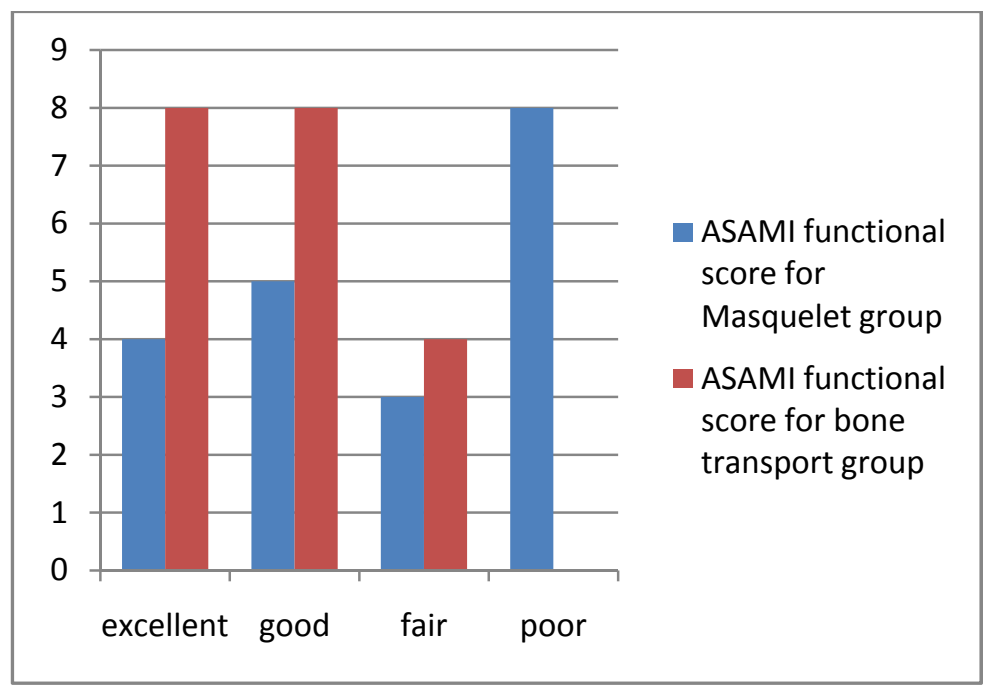

Diagram 3: bar chart for ASAMI functional score for both groups

Table 3 Comparison between (Masquelet and Bone transport) groups regarding bone union, ASAMI bony and functional score, and need for other operations showing significant difference.

\begin{tabular}{|c|c|c|c|c|c|c|c|}
\hline & & \multicolumn{2}{|c|}{$\begin{array}{c}\text { Group A } \\
\text { (masquelet) } \\
(\mathrm{N}=20)\end{array}$} & \multicolumn{2}{|c|}{$\begin{array}{c}\text { Group B } \\
\text { (bone transport) } \\
(\mathrm{N}=20)\end{array}$} & \multirow[t]{2}{*}{$\begin{array}{c}\mathrm{X}^{2} \\
\text { Fisher } \\
\text { Exact test }\end{array}$} & \multirow[t]{2}{*}{$\mathrm{P}$ value } \\
\hline & & $\mathrm{N}$ & $\%$ & $\mathrm{~N}$ & $\%$ & & \\
\hline \multirow{2}{*}{ bone union } & yes & 12 & $60.0 \%$ & 20 & $100.0 \%$ & \multirow[t]{2}{*}{10.00} & \multirow{2}{*}{$0.003 \mathrm{HS}$} \\
\hline & no & 8 & $40.0 \%$ & 0 & $0.0 \%$ & & \\
\hline \multirow{4}{*}{ ASAMI bony score } & excellent & 4 & $20.0 \%$ & 6 & $30.0 \%$ & \multirow{4}{*}{10.04} & \multirow{4}{*}{$0.002 \mathrm{HS}$} \\
\hline & good & 4 & $20.0 \%$ & 7 & $35.0 \%$ & & \\
\hline & fair & 4 & $20.0 \%$ & 7 & $35.0 \%$ & & \\
\hline & poor & 8 & $40.0 \%$ & 0 & $0.0 \%$ & & \\
\hline \multirow{4}{*}{$\begin{array}{l}\text { ASAMI functional } \\
\text { score }\end{array}$} & excellent & 4 & $20.0 \%$ & 8 & $40.0 \%$ & \multirow{4}{*}{10.72} & \multirow{4}{*}{$0.01 \mathrm{HS}$} \\
\hline & good & 5 & $25.0 \%$ & 8 & $40.0 \%$ & & \\
\hline & fair & 3 & $15.0 \%$ & 4 & $20.0 \%$ & & \\
\hline & poor & 8 & $40.0 \%$ & 0 & $0.0 \%$ & & \\
\hline \multirow{2}{*}{$\begin{array}{c}\text { Need for other } \\
\text { operation }\end{array}$} & yes & 9 & $45.0 \%$ & 3 & $15.0 \%$ & \multirow{2}{*}{4.29} & \multirow{2}{*}{$0.04 \mathrm{~S}$} \\
\hline & no & 11 & $55.0 \%$ & 17 & $85.0 \%$ & & \\
\hline
\end{tabular}

\section{DISCUSSION:}

Masquelet technique is a relative new technique used in the management of large bone defects. It is based on The formation of induction membrane ${ }^{8}$

Bone transport is considered an ideal treatment for large skeletal defects of any size without limb shortening. It allows for decreased need for casting post operatively, or bracing due to rapid corticalisation of regenerate bone and decreases need for bone grafting ${ }^{9}$
In our knowledge there is a little clinical prospective studies comparing Masquelet technique to bone transport technique of management of bone defects from the clinical \& radiological points of view, but there are some retrospective case series which analyzed outcomes of the Masquelet technique and segment transfer technique of management

In 2016, Ilaria $\mathrm{M}$, et $\mathrm{al}^{10}$, a systematic review and meta analysis study was done on the results of Masquelet technique in management of bone defects.IMT (induced membrane technique ) aims to achieve bone 
union and infection eradication, but persistence of infection or non-union was noted in $18 \%$ of cases necessitating reinterventions..Further studies are needed to demonstrate the role the patients' clinical features and IMT variants have upon achieving bone union and infection eradication.

In 2014, Tak $M$, et $\mathrm{al}^{11}$, study on Masquelet technique in post traumatic defect showed All patients demonstrated radiographic consolidation over the defect after treatment .

In 2012, Rigal S, et $\mathrm{al}^{2}$, The multicenter retrospective study on segment transfer combined 38 cases: 22 cases of initial diaphyseal bone defect and 16 cases of secondary diaphyseal bone defects, A mean 4.3 secondary interventions were required to obtain final union; most notably, a bone graft was necessary at the docking site for the segmental bone transport procedures.

In 2010, Claudio I, et $\mathrm{al}^{12}$, they present the results of treatment of 100 patients (72 men, 28 women) by the Ilizarov method of bone transport .. the total treatment time ranged from a minimum of 5 months to a maximum of 2 years. In 96 cases, bone transport led to normal neoformed bone.

All of the above studies recommended the need for prospective randomized comparative studies to compare the effect of Masquelet technique and segment transfer in bone defects

\section{In our study:}

\section{Radiological assessment showed:}

1- 8 cases with no evidence of healing .. all in group A (Masquelet group) and all these cases were secondary defects due to infected non union

2- 32 cases with complete healing:

- 12 cases in group A (Masquelet group)10 cases were primary posttraumatic defects and 2 cases were infected non union

- All cases in group B (bone transport group ).

\section{Clinical assessment showed:}

7 cases with recurrent infection

- 6 cases in group A ..

- 1 case in group B

We observed statistically highly significant difference between both groups in both radiological and clinical outcomes, which makes us in favor of segment transfer technique in management of large defects in lower limb.

As regards post-operative complications and the need of 2ry procedure, statistically highly significant difference appeared to be present between both techniques of management.

We also observed that type of graft used in Masquelet technique either iliac crest, non vascularized fibula, or even artificial bone subistitutes may affect the final result in masquelet case no 1 ( secondary proximal femur defect) where all conditions were classic and optimum for healing except for the artificial bone subistitutes which showed no evidence of calcification radiologically and clinically during removal, thus this may suggest that in that case bony subistitute was the main cause of failure??

Vascularized fibular graft in masquelet technique was done in our study in one case and the case was presented with infection and none union at one end and needed debridement and refreshening of edges, not totally satisfying result but still better than results of non vascularized fibular grafts which were sequestrated in most cases and showed no union, but still technically demanding lengthy operation.

Causes of failure of some cases of Masquelet may be due to mal positioned fibular graft as in cases no $6 \& 9$ where 
fibular grafts where put eccentric in case no 6 and penetrating the induced membrane in case no 9.

Lack of mechanical stability also may have a role in failure of some cases as in case no 9 where the pre existing osteomyelitis was severe and extensive involving the whole medulla leading to severely osteopenic bone and pins lost purchase in a rate higher than normal leading to early mechanical failure of the frame before healing.

We have only the experience of the Polymethylmethacrylate cement as spacer. Other materials have not yet been tried even in experimental studies.

We do not advise using antibiotic-loaded cement for 3 reasons:

1. The antibiotic may be inactive on the germ and is likely to increase biological resistance of germs.

2. Some active antibiotics can affect the characteristics of the membrane.

3. The absence of recurrent infection with cement without antibiotics is a good sign of healing.

Another common mistake is to think antibiotic-loaded cement is capable of treating bone infection and allowing a less important debridement.

Method of fixation in first stage Masquelet was either:

1. Monoplannar fixator: either due to lack of available permenant ring fixator on patient presentation or due to need for coverage by plastic surgeons.

2. Ring fixator (ilizarov frame) as a permanent method of fixation in pre prepared cases

3. LRS as a permanent method of fixation in pre prepared cases

4. internal fixation by plates in cases underwent repeated first stage
We also observed that Masquelet technique may have some fair results with small, metaphyseal and posttraumatic defects so we recommend to do Masquelet technique in these cases .

We also observed that bone transport can be also effective in dealing with soft tissue defects as well as bone defects, by doing composite transfer which is transporting both bone and soft tissue to fill bony and soft tissue defects, so limits the need for plastic coverage by muscle flaps or grafts

\section{Conclusion:}

Segment transfer technique is more reliable method with more expectable results for healing and infection eradication.

So, what about Masquelet technique ?? ..

Up to our study, Masquelet technique can be used in the following indications:

1. small defects

2. metaphyseal defects

3. posttraumatic and non infective defects

Otherwise, Masquelet technique's results are unexpectable and uncontrollable.

Bone transport remains the most versatile, effective treatment for complex cases involving extensive bony and soft tissue injuries., it is an important treatment method in circumstances with no acceptable alternatives. For optimal functional results, transfer to a specialist unit, patient selection, emotional, functional and social support is compulsory

Further studies are needed to demonstrate the role of Masquelet technique in achieving bone union and infection eradication.

further studies with larger samples and longer periods of follow up is required for additional assessment of the difference between the two methods of reconstruction 
as regard the union, function, complications as infection and fracture.

Also we recommend to do a systematic review of the case series that discuss the use of both techniques in reconstruction

There were certain limitations in our study:

$>$ First that we used plain radiographs \& CT scan to assess the bone remodeling, we still believe histological evidence of new bone formation in the defects is the gold standard and a prospective study to document this should be conducted.

$>$ Second different types of bone defects with different biological behavior treated with same kind of treatment were included.

$>$ Third, there were systemic factors such as age-related comorbidities and bone mineral density that may also influence the blood supply and bone healing were not included for analysis, which may be taken into account source of potential bias when interpreting results.

\section{REFERENCES:}

1. Karger, Claude \& Kishi, T \& Schneider, L. \& Fitoussi, F \& Masquelet, A-C. Treatment of posttraumatic bone defects by the induced membrane technique. Orthopaedics $\&$ traumatology, surgery \& research : OTSR 2012: 98. 97-102.

2. Rigal, S., Merloz, P., Le Nen, D., Mathevon, H. \& Masquelet, A.-C. Bone transport techniques in posttraumatic bone defects. Orthop. Traumatol. Surg. Res 2012 $\therefore$ 98, 103-108

3. Masquelet AC, Begue $\mathrm{T}$ The concept of induced membrane for reconstruction of long bone defects Orthop Clin North Am 2010;41:27-37.

4. Masquelet AC, Obert L. Chir Main Induced membrane technique bone defects in the hand and wrist. 2010;29(Suppll. 1):S221— 4. .

5. 5.. Anagnostakos $\mathrm{K}$, Fürst $\mathrm{O}$, Kelm J Antibiotic-impregnated PMMA hip spacers. (2006) Acta Orthop 77(4):628-637

6. Abbaspour, A., TakahashiM, Sairyo K , Takata $\mathrm{S}$,et al, Optimal increase in bone mass by continuous local infusion of alendronate during distraction osteogenesis in rabbits. 2009.Bone. 44:917-923.

7. Aronson, J., Shen XC, Skinner RA, et al, Rat model of distraction osteogenesis. $J$ Orthop Res. 199715:221-226.

8. Stafford PR, Norris BL Reamer-irrigatoraspirator bone graft and bi Masquelet technique for segmental bone defect nonunions: a review of 25 cases. Injury 41 Suppl 2:(2010) S72-77

9. .Bobroff GD, Gold S, ZinarTen year experience with use of Ilizarov bone transport for tibial defects. Bull Hosp Jt Dis (2003) 61(3-4): 101- 107

10. 10.Ilaria M, Lorenzo D, David A.G, Enrico $\mathrm{G}$, Sara S, Romanò L Masquelet technique: myth or reality? A systematic review and meta-analysis Injury Volume 47, Supplement 6, December 2016, Pages S68S76

11. Tak M, Tak W, Xin L, Christian F, et al"Masquelet technique for treatment of posttraumatic bone defects " The Scientific World Journal 2014 : vol. 2014710302.

12. 12.Claudio I, Antonio B, and Roberto A ,Bone transport using the Ilizarov method: a review of complications in 100 consecutive cases Strategies Trauma Limb Reconstr. 2010 Apr; 5(1): 17-22. 
بناء العظام المحفز عن طريق الغثاء (طريقة ماسكوليه) مقارنة بيناء العظام عن طريق النقل العظمى فى الفجوات العظمية الكبيرة بالطرف السفلى فئلى

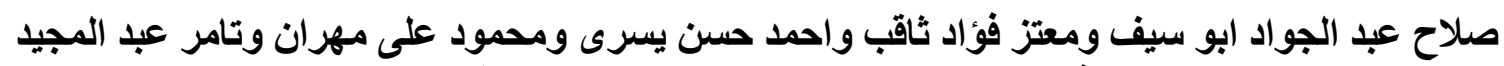

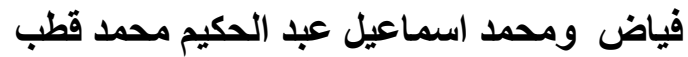

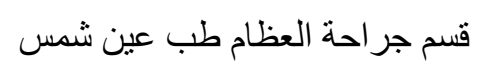

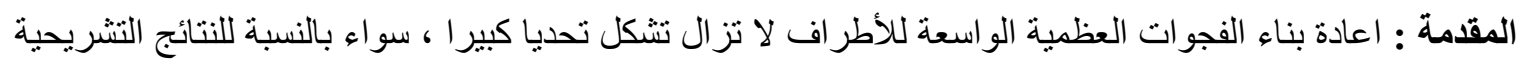

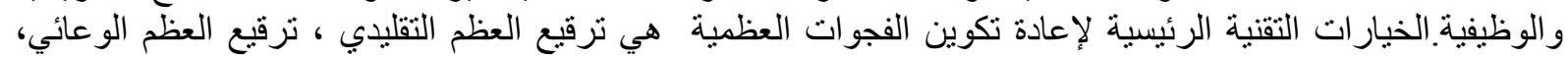
وتقنية الغثاء المستحث وتقنيات نقل العظم

الهاف من الاراسة : مقارنة نتائج طريقة الغشاء المستحث (ماسكوليه) طريقة النقل العظمى من حيث النتائج

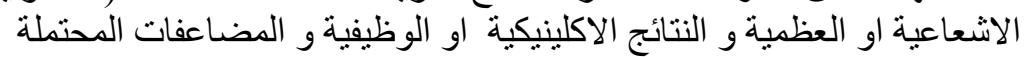

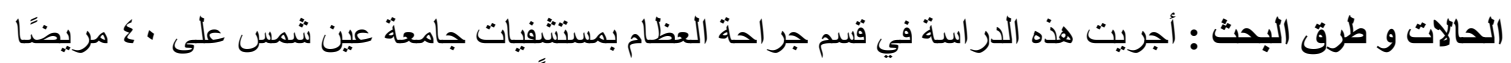

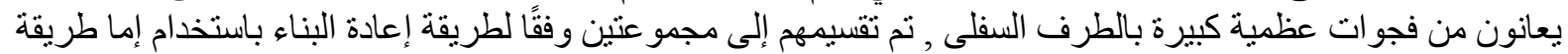

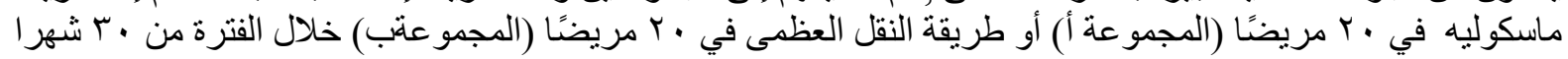

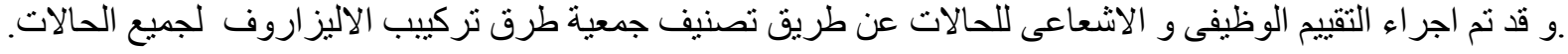

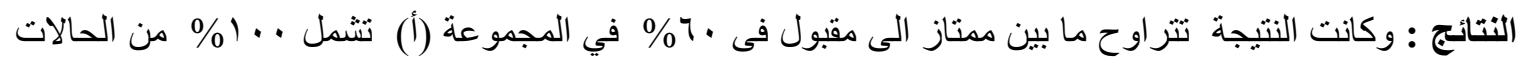

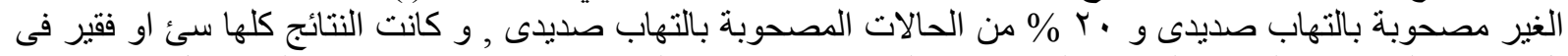

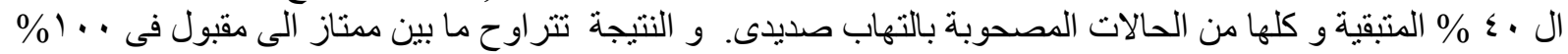
في المجموعة (ب).

الخلاصة : ختاماً، فنحن نوصي باستخدام أي من التقنتيتين فى حالات الفجو ات الغير مصحوبة بالتهاب صديدى ،ولكن فى حالات الالتهاب الصديدى نوصى باستخدام طريقة النقل العظىى . 